Chirurg 2014 · 85:640

DOI 10.1007/s00104-014-2794-y

Online publiziert: 18. Juni 2014

c) Springer-Verlag Berlin Heidelberg 2014
L. Haeder $\cdot J$. Jähne

Klinik für Allgemein- und Viszeralchirurgie, Schwerpunkt für endokrine und onkologische Chirurgie,

Diakoniekrankenhaus Henriettenstiftung gGmbH, Hannover

\section{Outcome von Patienten mit Ösophagusperforation}

\section{Ergebnisse einer Multicenterstudie}

\section{Orginalpublikation}

Biancari F, Saarnio J, Mennander A et al (2014) Outcome of patients with esophageal perforations: a multicenter study. World J Surg 38:902-909. doi:10.1007/s00268-013-2312-2

\section{Hintergrund}

Trotz verschiedener Behandlungsmethoden stellt die Ösophagusperforation immer noch eine hochakute Erkrankung mit einer signifikanten Morbidität und Mortalität dar. Die endoskopische Stentimplantation bietet einen im Vergleich zur operativen Therapie weniger invasiven sowie organerhaltenden Behandlungsansatz, dessen Vorteile bisher allerdings noch nicht bewiesen sind. Die vorliegende Multicenterstudie will daher das Früh- und Spätoutcome analysieren und zudem die Ergebnisse der chirurgischen sowie endoskopischen Behandlung miteinander vergleichen.

\section{Methoden und Ergebnisse}

Die Daten von insgesamt 194 Patienten mit einer Ösophagusperforation, die zwischen 01/2000 und 02/2013 in 9 internationalen Zentren behandelt wurden, konnten retrospektiv erhoben und anschließend insbesondere hinsichtlich des Outcomes statistisch ausgewertet werden. Die unterschiedlichen Behandlungsmodalitäten beinhalteten die konservative Therapie $(n=43)$, die Applikation von Endoclips $(n=4)$, die Stentimplantation $(n=63)$ sowie die chirurgische Therapie $(\mathrm{n}=84)$. Komorbiditäten inkl. Malignompräsenz, septisches Geschehen, Ausmaß und Lokalisation der Perforation wurden neben anderen Faktoren wie z. B. der chirurgischen Technik ebenfalls erfasst.

Eine univariate Analyse konnte diesbezüglich eine erhöhte Mortalität bei $\mathrm{Pa}$ tienten >70 Jahren, Begleiterkrankungen wie koronare Herzkrankheit, Niereninsuffizienz und vorbestehender Ösophagusneoplasie sowie einer Behandlungsverzögerung von $>24 \mathrm{~h}(22,7$ vs. $12,4 \%$; $\mathrm{p}=0,067)$ nachweisen. Insgesamt verstarben 43 Patienten (17,5\%). Die Behandlungsmethode wirkte sich nicht auf die Frühmortalität aus, allerdings war die chirurgische Therapie verglichen mit der Stentimplantation insgesamt mit einer etwas geringeren 30-Tage-Mortalität assoziiert (11,9 vs. 15,9\%). Die Frühüberlebensrate mit Organerhalt war dementsprechend in der Stent-Gruppe höher $(77,8$ vs. 56,0\%). Lediglich in einem Zentrum mit Stentimplantation als primäres therapeutisches Vorgehen $(n=26)$ konnte eine Mortalitätsrate von nur 7,7\% gezeigt werden.

\section{Diskussion und Fazit}

Analog zu einer früheren Arbeit der Autoren vermag auch diese Multicenterstudie bedingt durch die Heterogenität von Ursachen, Perforations- und Komplikationsschwere, relevanten Komorbiditäten sowie Zeitpunkt des Therapiebeginns keine signifikante Überlegenheit eines der dargestellten Thera- pieverfahren zu definieren. Zudem wird die Analyse durch den Präselektionsbias hinsichtlich minimal-invasiver Verfahren bei nur gering ausgeprägten Perforationen beeinflusst. Die Autoren gestehen ein, dass mindestens 500 Patienten in jeder Studiengruppe vorliegen müssten, um einen signifikanten Unterschied in der Frühmortalität herauszuarbeiten. Um den Effekt der endoskopischen Intervention mit dem Ziel des Organerhalts zu optimieren und die Mortalität signifikant zu senken, ist eine hohe Expertise mit einer ggf. chirurgischen Kotherapie unabdingbar. Es wird daher noch einige Zeit verstreichen, bis eine ausreichende und valide Datengrundlage vorliegt, die die optimale Behandlungsmodalität definiert. Hier müssen dann aber auch neuere Verfahren wie die EndoVac-Therapie mit inkludiert und analysiert werden. Unstrittig ist, dass die Komplexität dieser Erkrankung eine zeitnahe, individualisierte und interdisziplinäre Therapie erfordert.

\section{Korrespondenzadresse}

\section{Haeder}

Klinik für Allgemein- und Viszeralchirurgie, Schwerpunkt für endokrine und onkologische Chirurgie, Diakoniekrankenhaus Henriettenstiftung $\mathrm{gGmbH}$, Marienstr. 72-90, 30171 Hannover Lars.Haeder@ddh-gruppe.de

Interessenkonflikt. L. Haeder und J. Jähne geben an, dass kein Interessenkonflikt besteht. 